\title{
I TÉCNICA EN SU RELACIÓN CON EL ORGANISMO: LA HERRAMIENTA Y DESPUÉS*
}

\section{Technique in its relationship with the organism: the tool and afterwards}

\begin{abstract}
Resumen: el análisis parte de un texto en el que Hans Jonas presenta la herramienta prehistórica como un signo de humanidad; esta anunciaría el pensamiento técnico. Por lo demás, la filosofía de Jonas muestra las bases orgánicas de la libertad en lo que tiene de espiritual. En este sentido, precisamos que la técnica no aparece bruscamente como el producto de una consciencia reflejada: el pensamiento y la técnica mantienen vínculos cruzados de causalidad donde el hombre se descubre y accede a la representación de su acción. Estos dos tipos de miradas también son pertinentes para pensar la técnica moderna. Entre un pensamiento
\end{abstract}

\footnotetext{
" Robberechts, Bruno, "La technique dans son rapport à l'organisme: l'outil et après", Revue Philosophique de Louvain. Cuarta serie, T. 100, ํ3, 2002. pp. 360-384. [En línea] http://www.persee.fr/web/revues/home/prescript/article/ phlou_0035-3841_2002_num_100_3_7428. Agradecemos la amabilidad de Benoît Thirion de la redacción de la Revue philosophique de Louvain y al autor por permitirnos traducir y publicar este artículo.

${ }^{* * *}$ El abad Bruno Robberechts es ingeniero civil y doctor en filosofía. Profesor de filosofía y formador en el Seminario de Namur desde 1999. Director de la biblioteca del Seminario. Cura de las parroquias del sector pastoral de Fernelmont (Bélgica). Contacto: http://www.seminairedenamur.be/spip. php?auteur7\&secteur $=2$

**** Historiador, Docente Universidad de Antioquia, Medellín - Colombia, rodrigozapatak@yahoo.com.
}

Fecha de recepción: 9 de mayo de 2013

Fecha de aceptación: 9 de junio de 2013

\author{
Bruno Robberechts** \\ Traducción de Rodrigo Zapata Cano***
}

Thencion de Rodrigo Zap

abstracto a partir de la experiencia vivida y la intencionalidad propia de la acción, la mediación de los objetos técnicos aparece con una transparencia y una alteridad variables. Así pues, se trata de evitar que la atención prestada a la mediación técnica no oculte la acción en lo que la hace humana.

Palabras clave: Hans Jonas, pensamiento técnico, organismo, herramienta, acción, mediación.

Abstract: this paper is based on a text in which Hans Jonas presents prehistoric tools as a sign of humanity. These tools are said to announce technical thought. Moreover, Jonas' philosophy shows well the organic bases of freedom in its spiritual dimension. In this sense we emphasize that technology does not appear suddenly as the product of a reflecting consciousness: thought and technology have crossed links of causality, in which man discovers himself, and in which he has access to representation of his action. These two types of approach are also valid for our modern technology. Between abstract thought based on lived experience and the intentionality typical of 
action, the intervention of technical objects appears with variable transparency and otherness. The important thing, therefore, is to avoid that the attention devoted to technical intervention does not obscure the human ingredient present in action.

Keywords: Hans Jonas, technical thought organism, tool, action, mediation.

\section{INTRODUCCIÓN}

La herramienta prehistórica es testigo de la humanidad en sus orígenes. Un texto de Hans Jonas titulado "Outil, image et tombeau" (Jonas, 2000), nos la presenta como una diferencia antropológica. Sin embargo, como ser vivo el hombre se concede esta mediación por su relación con el mundo. Entonces se plantea una pregunta: ¿cuál es el lugar del organismo en la aparición de la herramienta? La perspectiva de mostrar una especificidad del hombre y el marco de la ética de Jonas donde la técnica amenaza la naturaleza podría ocultar aquí una mayor articulación, sobre la cual insiste la filosofía de Jonas: la que el cuerpo invita a reconocer entre los campos físico y espiritual y entre los caracteres físico y objetivo. Nos detendremos en esta pregunta crucial a propósito de la herramienta. Tomaremos de allí pistas para interpretar algunos aspectos de la técnica moderna.

En el texto mencionado anteriormente, Jonas no entra en el detalle de un estudio paleontológico. No haremos más que él. Si Jonas hace pensar en la inteligencia en la acción por medio de la utilización de herramientas, no nos limitaremos a este enfoque. Así, a través del fenómeno de la herramienta, validaremos la unidad del hombre, cuerpo y espíritu, es decir, el juego recíproco entre ambos. Nuestros diferentes enfoques de la herramienta corresponden a un despertar más o menos grande de la acción técnica, en una gradación que va de los automatismos que porta el aparatado sensorio-motor hasta las secuencias de gestos que reclaman una real atención y que son susceptibles de un mejoramiento inteligente. La evolución somática y mental que le corresponde dará un marco al problema del hombre. La herramienta parecería mostrar que el hombre, por su inteligencia, se libera de lo que tiene de orgánico. Antes de esto, será necesario subrayar cómo la herramienta, incluso sin haber salido directamente del organismo, supone ciertas características de este. Desde luego, los datos cambian cuando la ciencia interviene y cuando las condiciones culturales y económicas contribuyen a un progreso tecnológico sostenido ${ }^{1}$. ¿En qué se convierte entonces la referencia dada por el organismo para el uso de la técnica, cómo marca su presencia en la relación del hombre con el mundo si los artefactos remplazan el trabajo corporal 0 , por lo menos, si se deben integrar auxiliares artificiales en su acción? Una breve introducción a la filosofía de la vida de Jonas y al problema de la herramienta nos ayudarán a entrar en este cuestionamiento.

\section{EL ORGANISMO Y SU APERTURA A UNA DIMENSIÓN ESPIRITUAL: LA LIBERTAD INHERENTE A LA VIDA}

La filosofía de Jonas se preocupa por mostrar, en la vida, la dimensión espiritual introducida por una $u$ otra forma de libertad. De este modo, para Jonas la herramienta es reveladora (en un estadio de su historia que es preciso discutir) de un trabajo espiritual en el seno de la vida orgánica. Para comprenderlo mejor, es necesario poner en evidencia una articulación que

\footnotetext{
${ }^{1}$ Jonas lo señala al describir la historia de la técnica en el siglo XVII (Jonas, 1974). Hans Jonas tiene un doble enfoque de la técnica. Muchos lo conocen por haber extendido la responsabilidad a las futuras generaciones, a causa del potencial destructivo de nuestras actividades técnicas. Esto tiene que ver con la técnica moderna donde prima una lógica del desarrollo y una racionalidad científica. También reconoce la técnica en su papel durante el episodio más largo de su historia enfocada hacia la herramienta. Al comienzo, la técnica es "el empleo de herramientas y de equipos artificiales para el comercio de la vida" y es al mismo tiempo "su descubrimiento original, su fabricación repetida a partir de allí y en ocasiones también un añadido al arsenal existente" (Jonas, 1987 , p. 17).
} 
habita como un proyecto la interpretación filosófica de la vida, que Hans Jonas hace en The Phenomenon of life (Jonas, 1963). La filosofía de la vida de Jonas comprende una filosofía del organismo donde se muestra una apertura hacia el espíritu. El sentido que se le da aquí al organismo y al espíritu no tiene nada de limitativo: el organismo remite a la célula más rudimentaria, así como al organismo humano; el espíritu no es el atributo de las más altas facultades intelectuales. Jonas lleva al lector a descubrir que lo espiritual no se vislumbra como un campo limitado en el hombre: en la escala de lo viviente, del menos evolucionado al más evolucionado, encontramos facultades cada vez más espirituales hasta devenir, en el hombre, consciencia reflexiva y libertad de elección. Estamos invitados a reconocer, en esta clase de enfoque, que el organismo más simple es actor, por medio del metabolismo y, de alguna manera, hace la elección de la vida, la elección de afirmarse así mismo en el encuentro de la opción opuesta: el no-ser. De nuestra parte, antes de decir que la herramienta marca una diferencia antropológica, en unión con la aparición de la inteligencia y una actividad espiritual, queremos subrayar el hecho de que el organismo lleva esta dimensión espiritual.

\section{LA TÉCNICA, LIBERTAD DISTINTIVA DEL HOMBRE, PARO ARTICULADA A LAS LIBERTADES PRESENTES EN LA VIDA ANIMAL}

Nos equivocaríamos al fundamentar toda la técnica en la inteligencia. La técnica se aprecia en el comportamiento de algunos animales sin que tengan una consciencia técnica. Creemos que los animales superiores muestran una técnica primitiva. Esta responde sin duda muy directamente a las necesidades de la vida, pero toma distancia con las respuestas más simples a estas necesidades. Esta técnica descansa en facultades que Jonas muestra como otras formas de libertad que aparecieron con la vida. Para los animales insiste en la locomoción, la percepción y la emoción. Precisemos que la emoción se comprende aquí a partir de sus formas mínimas, por ejemplo cuando una tensión permite proseguir un comportamiento poco elaborado, aun cuando sea desencadenado por una simple señal y no por una verdadera elección. Detengámonos en la opción tomada por Jonas de introducir aquí la dimensión de un apetito, algo emparentado con el deseo. Jonas está atraído por la experiencia humana de la emoción, que es especialmente más rica. La imaginación o la inteligencia que permiten este enriquecimiento dejan adivinar que la técnica, en lo que tiene de propiamente humano, no es otra cosa que una búsqueda titubeante de medios para vivir o sobrevivir.

Jonas prefiere explicar la vida animal por medio de la vida humana. Por muestra parte, queremos integrar las riquezas encontradas en el animal en nuestro enfoque del hombre. De esta manera subrayamos, por ejemplo, que el uso de una herramienta depende de un buen ajuste entre la motricidad y la percepción. En este sentido, el carácter técnico no está solo en la presencia de una herramienta, sino además en las aptitudes del organismo humano ${ }^{2}$. Quisiéramos aclarar el vínculo entre tales aptitudes y la inteligencia presente en la técnica leída a través de una perspectiva más moderna. La técnica parece marcar una diferencia cualitativa con relación a los medios naturales de los que el viviente dispone para vivir. No obstante, se debe precisar mejor cómo es la técnica en la prolongación de las facultades de la vida animal.

\footnotetext{
${ }^{2}$ Esta perspectiva es la que, por ejemplo, propone Tim Ingold en "Tool-Use, sociality and intelligence" (Ingold, 1983, pp., 433-436), la que nos invitará a continuación a integrar a la herramienta mucho más en capacidades indisociablemente corporales y mentales.
} 


\section{DOS ENFOQUES DE LA HERRAMIENTA: PENSAR LA HERRAMIENTA Y VIVIR LA ACCIÓN. CÓMO LA CAPACIDAD EIDÉtICA, MENTAL Y CORPORAL INVITA A ARTICULARLAS}

La filosofía de la vida de Jonas muestra algunos indicios de la relación de un organismo con el medio que lo rodea. ¿Qué sucede, en esta relación que es la vida humana, cuando un hombre, por medio de su organismo, utiliza una herramienta? Sin querer marcar la diferencia que sería buscar la especificidad del hombre allí, insistimos en el lugar de la herramienta en un gesto técnico en el cual el hombre emplea su fuerza y debe ajustar su acción a lo que ve. Este gesto también está motivado por la intensión que apunta a la acción. Por el contario, si el análisis se dirige ante todo a la herramienta como objeto, la interrogación implica la manera por la cual esta fue elaborada, concebida o pensada. De este modo, se presentan dos enfoques de la herramienta: ya sea pensar la herramienta en sí misma o remitirse a los gestos en la que interviene. En la primera opción, se aborda el pensamiento que permite concebir una herramienta, suponiendo que se le reconozca el estadio de evolución de la técnica. En la segunda opción, la herramienta aparece como un producto de la práctica y no es disociable de esta parte de la vida que es la acción técnica.

\section{LA HERRAMIENTA COMO UN MEDIO CONCEBIDO PARA UN FIN}

En el marco del artículo "Outil, image et tombeau", Jonas quiere abordar las huellas de la vida de nuestros ancestros sobre la base de los indicios que nos legaron. Así pues, la interpretación de la herramienta no puede estar más abierta, y es por alusión a una forma de pensar que Jonas eligió la herramienta como una marca de humanidad ${ }^{3}$. Para Jonas, hablar de una herramienta supone que esta tenga una finalidad, que un hombre le haya dado una finalidad (Jonas, 2000, p. 80). En esta óptica, la herramienta es un objeto que ha sido pensado "en una relación del fin al medio y de la causa al efecto" (Jonas, 2000, p. 80). ¿Nos enfrentaríamos con una racionalidad semejante a la de un técnico o un ingeniero de la actualidad? Jonas matiza su posición al abrir el problema de la intencionalidad: "es posible que no sepamos qué fin determinado tenía una herramienta, pero sabemos que tenía uno" (Jonas, 2000 , p. 80). No continuamos aquí en la finalidad propia de la vida animal que Jonas describe por medio de la facultad de la emoción, pues precisa su tesis al indicar en la herramienta el anuncio del pensamiento presente en la técnica y la fisica ${ }^{4}$. El punto de vista es problemático, puesto que nada dice que Jonas trate sobre herramientas presentes en una época en que la evolución técnica ya se había liberado de la evolución del organismo de los primeros hombres. ¿Sería necesario acortar considerablemente la historia de la herramienta humana y asociarla a una capacidad de pensar abstracta y técnicamente una relación del medio con el fin buscado? La pregunta permanece abierta $^{5}$. Pero no impide tomar un poco de distancia con relación a la conceptualización de la técnica

\footnotetext{
${ }^{3}$ Esta opción de Jonas no le exige prestar atención a los elementos de los que da testimonio la antropología cultural. La libertad de interpretación, que debe remediar la pobreza de los datos brutos, no significa una ignorancia de Jonas, por ejemplo en lo que atañe a la vida social o las facultades sensorio-motrices. Otros textos, como "Technik, Freiheit und Pflicht", sugieren la importancia de la voz y de la comunicación para hacer de la sociedad "un tema permanente de la memoria creciente" (Jonas, 1993, p. 94). En otros textos de Jonas se desarrolla además la riqueza de la percepción en el hombre y del comportamiento que se apoya en ella. (Véase, por ejemplo, "The nobility of Sight" en The Phenomenon of life (Jonas, 2000).

${ }^{4}$ Jonas sitúa estas formas de pensamiento en la prolongación del pensamiento en acción con la herramienta.

${ }^{5}$ Al señalar la herramienta como un rasgo distintivo del hombre, la tesis de Jonas plantea que la herramienta no tiene que ver con el organismo. En efecto, no se transmite de forma hereditaria. Su evolución no está ligada estrictamente a la evolución somática del hombre. Así, Jonas nos ubica en el estadio de la técnica situado después de lo que Leroi Gourhan llama el segundo nacimiento del hombre, en el momento en que la evolución del utillaje se desliga con respecto a la evolución de algunos factores morfológicos humanos, en particular la capacidad craneana. Habrá que esperar al homo sapiens.
} 
moderna, para reunir las características propias de la intencionalidad de la acción y la apertura de un pensamiento arcaico en la representación de las formas:

Hemos elegido oponer dos enfoques de la herramienta. La posición teórica alcanzada, como lo ilustra un pasaje del Principio de responsabilidad, para definir la herramienta según su finalidad: la finalidad determina la esencia de la herramienta, hace parte de ella, de tal modo que esta esencia determina la existencia de la herramienta y es la causa de su devenir (Jonas, 1990, pp. 80-81).

Jonas no llega a retomar esta concepción a propósito de la herramienta prehistórica. Y su filosofía abierta a fines naturales, es decir, no representados, ino nos autorizaría a imaginar una especie de pensamiento no reflexivo de la herramienta? Pensar un fin es diferente de pensar el vínculo en sí mismo entre la herramienta y este fin. Así pues, proponemos buscar cómo una finalidad primero más flexible pudo llevar a concebir, progresivamente, la herramienta con vistas a un fin. Además, pensamos que esta pregunta puede aclarar la relación que existe para el hombre en la actualidad entre la inteligencia técnica y el organismo y su acción. Formulemos de otra manera nuestra tesis: en un primer momento, una finalidad natural sería intervenida para determinar lo que es la herramienta, a través de la acción del hombre, pero sin que el hombre se dé cuenta de ello completamente. Con el tiempo, con la aparición de nuevos comportamientos, habría un lugar para la utilización de una herramienta en los tanteos de la vida para responder a sus necesidades. La complejización del comportamiento animal se muestra por ejemplo cuando los simios utilizan ramas o piedras. El hombre se ha liberado de comportamientos similares en lo que tienen de innato (o que se reactivan con un simple aprendizaje). Pero esto no quiere decir que la herramienta humana no proceda de un trabajo de búsqueda que conviene ubicar en lo activo, por así decirlo, de la vida. El hombre ha continuado este trabajo por su cuenta. El aprendizaje y la variación de las formas de las herramientas están asegurados no solo a nivel de la herencia, sino a nivel de la vida social del hombre. Esta memoria colectiva es muy diferente de una inteligencia abstracta individual reactivada cada vez. Jonas evoca esta memoria colectiva cuando habla de la posibilidad de un descubrimiento afortunado de la herramienta, seguido de la intervención de la facultad eidética para imitarlo. Volveremos sobre esto al subrayar cómo la herramienta está vinculada con un segundo rasgo de humanidad: la imagen. La imagen, que atestigua la facultad que permite separar las formas a partir de la percepción, muestra algunas condiciones del pensamiento técnico que se establecen con la vista en el hombre. De esta manera, encontramos en la filosofía de Jonas que la percepción (en particular la vista) sobre la que descansa la acción, tiene características que influyen sobre el pensamiento de un hombre.

\section{PENSAR LA ACCIÓN DONDE INTERVIENE LA HERRAMIENTA}

Al tomar ahora una más justa medida de la intención que conlleva en el hombre la acción, buscamos aclarar una especie de pensamiento, que implica la significación que toma para él el uso de la herramienta en la acción.

Esta significación depende de una cierta consciencia del actuar. Cuidémonos de no dejarnos engañar por un pensamiento muy abstracto de la técnica que ocultaría lo que induce a recurrir a este. Antes de acceder a una compresión técnica de la acción, lo que le da sentido es lo que intentamos alcanzar como seres vivos. Lo demuestra el hecho de que al usar una herramienta, nuestra atención no permanece focalizada en esta herramienta como medio para nuestra acción. 
Podemos apropiarnos de una herramienta. Después de un periodo de aprendizaje que exige mucha atención, la herramienta deja de considerarse por sí misma; se olvida en la acción. El examen de la herramienta prehistórica incita a plantear el problema del comportamiento técnico, no solo como concepción razonada de una herramienta, sino además como esquema de gestos dirigido por una intencionalidad y construido en una adaptación para una acción más eficaz. La herramienta interviene en esta adaptación y el hombre se la apropia.

Ricoeur habla de un vacío de la intención que la acción viene a llenar (Ricoeur, 1967, p. 191). El vacío señala que no existe representación de la acción, en el sentido en que la acción se pensaría de antemano. Para explicar mejor esta intención, Ricoeur habla de una intencionalidad práctica como "acción que "colma" un proyecto" (Ricoeur, 1967, p. 191). Parece que la acción solo se piensa globalmente, a diferencia de un proyecto, de una representación de la acción. Encontramos aquí, en una explicación de nuestro actuar, una rejilla de lectura para pensar el gesto donde está presente una herramienta:

\footnotetext{
Desde el punto de vista de quien actúa, la herramienta en la mano, la acción atraviesa como un único mediador orgánico el órgano prolongado por la herramienta; la atención está principalmente en el pragma y secundariamente en la pareja indivisible órganoherramienta, que se percibe como una extensión del órgano (Ricoeur, 1967, p. 199).
}

El organismo orienta el comportamiento en el modo propio de la acción. Anticipa y ajusta percepciones y gestos, aun cuando intervenga una herramienta. Es una característica de nuestra apertura al aprendizaje y a la reorganización de los componentes de nuestro comportamiento. Reconocer una herramienta como concebida para un fin supone salir del modo propio de la acción. Así pues, al representarse la acción, aparece una finalidad que ya no está primero vacía, sino objetivada.

Evocar la técnica moderna no supone evacuar la finalidad de un ser dirigido por una emoción. Desde luego, haylugar para un pensamiento teórico que explica la práctica. Pero el uso de este pensamiento teórico deja el lugar, en las relaciones más prácticas de la vida, a la manera por la cual se asumen las posibilidades técnicas, vinculadas con las del organismo. Así pues, el organismo da el sentido, de algún modo, a la acción y la significación de la herramienta. Pensemos en las operaciones que consisten en hacer cuerpo con un objeto técnico, y donde este objeto se percibe primero en sus reacciones en el transcurso de una acción orientada hacia un fin.

Seguir la evolución de la herramienta en el filo de las edades de la humanidad es seguir al mismo tiempo la manera por la cual el pensamiento, ya presente como memoria y anticipación operatoria, se autonomiza al tomarse como objeto para enseguida dar la impresión de dirigir el cuerpo como desde el exterior. La herramienta contribuye a este proceso, en tanto suscita una atención a cualquier cosa exterior al cuerpo y a algo diferente del fin orientado por el cuerpo. Y seguramente, desde el momento en que fue preciso preparar o concebir una herramienta, se impone la atención sobre el fin por la que se hace. En los estadios primitivos de la técnica, era necesario dejar el lugar a una especie de intuición de soluciones, que de algún modo procedía de la enseñanza que proporciona la manipulación de los objetos. Por ejemplo, se trata de sentir cómo un guijarro se deja cortar para luego obtener un mejor uso de él. ¿Es para dar una forma cuya eficacia se comprende o simplemente para seguir un modelo que ya está presente? Antes de elegir entre las dos posibilidades, conviene mostrar, sin poder explicar el origen absoluto de la técnica, cómo las relaciones de manipulación llevan a una toma de consciencia. En 
resumen, podemos decir que la herramienta emerge de una práctica todavía animal cuando su forma es muy importante culturalmente para que se trasmita, y beneficia un saber-hacer que evoluciona hasta una libertad con relación a los modelos, una libertad como factor de la novedad.

Este rodeo por una concepción más práctica de la acción nos invita a mirar de otra manera sobre la posición de Jonas. Si nos remite al pensamiento del hombre en la actualidad para explicar la interpretación de la herramienta prehistórica ipor qué no aprender, al contrario, de las lecciones de una técnica más antigua, por qué no explicar nuestra acción, no solo por una representación de los medios que se otorga, sino además por las determinaciones, incluso las soluciones, donde el organismo desempeñaba un papel más importante?

\section{UTILIZAR O VER LA HERRAMIENTA}

Al suponer que las máquinas dejan en la actualidad en nuestra acción un lugar para una manipulación muy directa de la materia con las herramientas, podemos redescubrir posiciones de nuestro organismo que nos orientan en la acción, así como ellas orientaron a nuestros lejanos ancestros. En la práctica artesanal, tanto en la actualidad como en otras épocas, se trata de adquirir automatismos. Conviene subrayar aquí la importancia de estos dos sentidos que son la vista y el tacto. En una descripción de los diferentes sentidos, Jonas muestra muy justamente cómo la vista neutraliza la dinámica de la percepción, esta dinámica tan importante en el tacto. Ahora bien, también nos indica que la vista es un sentido preeminente en el hombre. Esto se debe a que en el hombre encuentra su lugar un pensamiento abstracto, un pensamiento que toma distancia con relación a la acción ${ }^{6}$. Caracterizar al hombre por medio de una técnica pensada, calculada, es ser coherente con un enfoque del conocimiento humano donde prevalecen las informaciones que proceden de la vista. Pero, con la condición de mostrar cómo ocurre, y también con la condición de no aislar la vista de los otros sentidos, pues esto iría en contra de lo que es nuestro organismo. Jonas subraya que el conocimiento, considerado también desde el modo de la visión, está informado por una experiencia corporal ${ }^{7}$. De esta manera, nos muestra que utilizar una herramienta supone más que informaciones visuales, pues el hombre aprende por medio de su acción y una relación práctica con las cosas. La experiencia corporal provee nociones tan importantes para la técnica como las de causalidad o fuerza, donde la vista no puede ser directamente la fuente ${ }^{8}$. Adivinamos la importancia de la noción de causalidad para concebir características técnicas como lo cortante, las posibilidades de perforar, la resistencia, o para comprender que una cierta masa representa una inercia muy útil en la percusión. El hombre tuvo que experimentar con las propiedades de diferentes materias antes de concebir la herramienta. ¿La asociación entre una

\footnotetext{
${ }^{6}$ Encontramos esta presentación en el artículo "The Nobility of Sight" donde esta nobleza consiste en ir hacia la objetividad y la teoría (Jonas, 1963, pp. 135-147). Sin embargo, es necesario anotar que si "la vista es el más libre", es "al mismo tiempo el menos "realista" de los sentidos" (Jonas, 1963, p. 147).

${ }^{7}$ La "fenomenología de los sentidos" desarrollada en The Phenomenon of life conserva lo que Jonas desarrolla en el apéndice "Sight and Movement", que sigue el artículo "The Nobility of Sight", o todavía en el artículo "Sight and Thought), que continúa en los Philosophical Essays. En efecto, Jonas muestra aquí la importancia de la motricidad y la sinestesia.

${ }^{8}$ Según Jonas, el pensamiento que permite pensar verdaderamente la herramienta reposa en la experiencia de una fuerza, en una experiencia del cuerpo y no, como en Kant, en una categoría a priori. La noción de fuerza está ligada aquí, pues permite experimentarla en virtud del principio de la acción y de la reacción. La previsión del comportamiento de un trozo de materia descansa en una experiencia que ha puesto al hombre en interacción con la materia, que le ha permitido experimentar su resistencia a los continuos choques y fuerzas, su inercia y su peso (véase por ejemplo el apéndice "Causality and Perception" en The Phenomenon of life). Asimismo con la distancia de la abstracción, preparada por el carácter menos comprometido de la vista, el hombre integra datos de la experiencia corporal para entrar en una relación práctica. Algunas técnicas reposan en abstracciones; esto no impide a quien dirige una acción técnica encontrar las características de la acción.
} 
propiedad de la materia y su uso "tecnológico" debe pasar obligatoriamente por un pensamiento técnico abstracto? La finalidad de la acción está soportada por una cultura técnica que no solo comprende este conocimiento de las propiedades de la materia, sino además soluciones ya existentes que proceden de la sofisticación progresiva de las soluciones. Las propiedades de la materia que se buscan una y otra vez para fabricar una herramienta no son primero pensadas, sino experimentadas. Son informaciones que impregnan el pensamiento teórico, para comprender como el conocimiento que, como la vista, neutraliza la dinámica de la relación con las cosas. ¿Limitarse a un pensamiento abstracto para la herramienta, no sería dar un rodeo?

\section{LA APTITUD DE REPRESENTARSE LAS FORMAS}

El acontecimiento del pensamiento, en el sentido fuerte de la historia de la herramienta, supone que el vínculo entre la forma y la función de esta última sea un objeto de este pensamiento. ¿Con base en la finalidad que le da a la herramienta, cuándo le dio el hombre una forma que se le adapta? Ante todas las hipótesis que suscita esta pregunta, podemos partir de algunos elementos de respuesta, entre los cuales existe la ruptura del paralelismo de los ritmos de evolución de los parámetros que atañen respectivamente a las técnicas de obtención de las herramientas y los datos morfológicos relativos al cerebro (Leroi Gourhan, 1966, p. 195). Pero las disposiciones fisiológicas favorables al pensamiento no informan necesariamente sobre su uso por una consciencia técnica reflexiva. El sistema nervioso no es solo el lugar del pensamiento abstracto, sino también el de la coordinación del conjunto de los gestos del organismo y del tratamiento de las informaciones sensoriales. Su sofisticación es igualmente indispensable para una vida social donde es posible un aprendizaje de la técnica, donde se transmite una cultura de la herramienta ${ }^{9}$. Para esta transmisión, factor descrito profusamente por Leroi Gourhan (Leroi Gourhan, 1945), Jonas muestra la importancia de la capacidad de representación de las imágenes. Esta capacidad atestiguada por antiquísimas pinturas también está presente en las herramientas. Con respecto a estas últimas, dice Jonas:

\begin{abstract}
La forma que se presenta a la imaginación se impone a la materia; se percibe de manera intuitiva en el modelo terminado y se reproduce en su multiplicación. Esto supone la fuerza eidética de la imaginación y el control eidético de la mano (y de cualquier movilidad voluntaria). Ya estamos en la facultad imaginativa del hombre, la cual no coincide simplemente con el "pensar", pero le sirve de zócalo y complemento a través de su imaginación lúdica (Jonas, 2000, p. 66) ${ }^{10}$.
\end{abstract}

De este modo, abordamos una noción esencial de la antropología de Hans Jonas: la capacidad eidética (Jonas, 1963).

La capacidad eidética le permite al hombre representar formas. Es una apertura al simbolismo y a una representación de la realidad en la imaginación. En un texto sobre la imagen, Jonas señala cómo el control eidético de la movilidad es una condición para cualquier tecnología (Jonas, 1963, p. 172). Para dibujar una imagen o fabricar una herramienta, la voluntad no solo debe desencadenar una acción:

\footnotetext{
${ }^{9}$ Recordemos la siguiente sugerencia de Hervé Kempf en un artículo sobre el nacimiento de las herramientas: "Si lugar a dudas, es necesario ponderar el punto de vista tecnicista del prehistoriador -sesgado por la falta de datos a su disposición- y plantear más fuertemente la pregunta del vínculo entre la utilización de la herramienta y la organización social" (1997, p. 94).

${ }^{10} \mathrm{El}$ vínculo entre la herramienta y la imagen no solo se orienta hacia el trabajo de la imaginación en un sentido técnico. Los estudios sobre el pensamiento técnico darían una explicación útil aquí. Como en el caso de las pinturas rupestres, es posible que valga una interpretación para la forma de las herramientas donde interviene una práctica ritual. En la "Filosofía de las formas simbólicas", Ernst Cassirer comenta algunos ejemplos ofrecidos por la etología: "De una manera general, encontramos repartida sobre la tierra la creencia en un poder mágico que poseerían algunas herramientas de trabajo, algunos instrumentos y algunas armas (Cassirer, 1972, p. 250). Además, muestra que el uso de la herramienta es un momento decisivo donde el hombre, con un poder a la vez mágico y técnico sobre la naturaleza, aprehende progresivamente su acción.
} 
debe dejar lugar a lo que permite la precisión de los diferentes gestos. Percibimos aquí el estrecho vínculo entre control de la mano y representación de las formas. Para tallar herramientas prehistóricas, también intervienen obstáculos directamente relativos a las propiedades de las piedras: una piedra no se deja tallar tan fácil como escribir sobre el papel. De esta manera, en la herramienta encontramos una "información exteriorizada": Frank Tinland, siguiendo a Leroi Gourhan, señala que aquí interviene no solo la materialidad del objeto que se acaba de evocar, sino además "las reglas de su producción de acuerdo a un estereotipo siempre identificable por su referencia a una cultura de origen" y "el conjunto tecnológico que provee los medios de su producción al artesano" (Tinland, 1977, p. 177). Conviene subrayar la dimensión simbólica de la referencia de la herramienta en un contexto cultural: en este sentido, sería posible buscar explicitar además, por ejemplo, lo que la herramienta quiere decir en cuanto a la relación entre los grupos humanos o en la relación de un hombre con la naturaleza (Wynn, 1983, p. 402; Ingold, 1983, pp. 441-442).

Antes de llegar a decir cómo la herramienta se hizo para algo y cómo su forma es representativa de una cierta intencionalidad de la acción que la utiliza, es preciso desplazar esta intencionalidad en el sentido de la capacidad eidética del hombre, en particular con su dimensión práctica ligada a la motricidad y a la experiencia corporal de los diferentes gestos.

Jonas muestra en la herramienta una libertad con relación a un comportamiento determinado por el cuerpo. Es exacto decir que esta libertad está en oposición a una práctica transmitida de forma hereditaria. Pero, la herramienta está unida al organismo en el sentido en que el cuerpo asimila los gestos técnicos, porque la talla de una herramienta pasa por los gestos que el cuerpo registra. De este modo, el cuerpo se encarga de acceder, a través de automatismos, a lo que está enfocado en la acción. En función de la complejidad de los comportamientos, se requiere la memoria colectiva 0 un pensamiento técnico individual para completar los automatismos corporales. Entonces se plantea una pregunta: isi la experiencia y el aprendizaje para el organismo son condiciones para la realización de herramientas, si la vista y la motricidad cooperan en la facultad eidética que permite reproducir las formas que una cultura conserva, no tenemos aquí las condiciones para que nazca un verdadero pensamiento técnico? Así, la capacidad eidética bien podría ser, en definitiva, el elemento distintivo de la técnica humana y, por ende, la marca distintiva del hombre que concibe y utiliza la herramienta.

\section{EVOLUCIÓN DEL HOMBRE Y LA HERRAMIENTA}

En un texto titulado "Technique, liberté et devoir" (Jonas, 1993), Jonas recuerda las particularidades de la especie humana que interpreta como las raíces biológicas de la libertad. Con la posición bípeda, la mano libre para la manipulación de las cosas, la mirada frontal y la voz infinitamente modulable, esta libertad va en el sentido de la herramienta, la comunicación y el pensamiento. Así, Jonas destaca las condiciones anatómicas que permiten la herramienta. ¿Solo podemos hacerla en condiciones pasivas? ¿En qué medida el cuerpo no ha contribuido a hacer aparecer la herramienta y no ha servido de modelo para la herramienta? 0 bien ila herramienta no ha permitido revelar el comportamiento ya técnico del organismo? Jean Piveteau plantea la siguiente pregunta en un artículo sobre el nacimiento de la reflexión: "ien vez de ser un resultado de la hominización, no es la herramienta más bien uno de sus factores?" (Piveteau, 1962, pp., 49-51). Esto llegaría a ilustrar la fórmula de Anaxágoras, quien decía que el hombre es inteligente 
porque tiene manos ${ }^{11}$. Conviene precisar de qué tipo de causalidad se trata. La práctica del hombre tendría influencia sobre sus aptitudes mentales:

140
Por intermedio de la herramienta se debieron producir una serie de reacciones correlativas, pero alternativas y desfasadas en el tiempo, que poco a poco los ha perfeccionado a ambos para alcanzar la liberación del poder reflexivo y la mutación del pre-hombre en hombre (Piveteau, 1962, p. 51).

El dato primero sería una cierta práctica instrumental, que fue posible por la mano liberada de la locomoción. Pensamos que la práctica instrumental se ha vuelto cada vez más fina por una serie de adaptaciones y tanteos cuyos éxitos se conservaron. El hombre debía encontrar con qué representarse su acción. La herramienta contribuyó con su exterioridad. La herramienta interviene como causa en un juego de espejos, donde el hombre deviene un hombre inteligente.

\section{LA MAYÉUTICA DE LA HERRAMIENTA}

Bernard Stiegler explicita, en su libro La technique et le temps (Stiegler, 1994, p. 167), el vínculo entre la herramienta y el pensamiento que ha determinado la evolución técnica. Hace el análisis de las tesis que relacionan el nacimiento del hombre con la técnica primitiva. Habla de una mayéutica realizada por la herramienta. Muestra cómo el utillaje y el córtex se diferencian juntos, como en un juego de espejos. La herramienta es una especie de memoria. De esta forma, el hombre "está guiado por lo que diferencia, incluso si descubre y se diferencia aquí, en una palabra, si inventa y encuentra su imagen aquí" (Stiegler, 1994, p. 167). Lo que guía a Stiegler es evitar un cambio radical de comportamiento técnico que intervendría

${ }^{11}$ Citado por J. Piveteau en (Piveteau, 1962, p. 51). causado por una entrada brusca en la escena de una inteligencia abstracta, en comparación con la escala del tiempo de la evolución somática. La anticipación operatoria es una noción importante al respecto, pues está presente en cualquier gesto técnico. Es necesario articularla a la anticipación que permite diferenciar el utillaje y adaptarlo mejor. Opera en la percepción, sobre todo por una referencia a la motricidad y por una especie de reactivación de movimientos pasados.

Esta primera anticipación interviene en el uso que hace el organismo de una herramienta que, de algún modo, da una primera comprensión de la acción. Stiegler muestra que esta anticipación operatoria no se puede disociar de la anticipación que permite la evolución de las herramientas. La reflexión que corresponde a la posibilidad de articular los componentes de la acción en el tiempo, por una especie de interioridad, no se opone al "conocimiento del organismo" de gestos asimilados y, por ende, interiorizados.

Al volver a la importancia de la propioceptividad y al evocar la reflexión, somos conducidos a los dos enfoques de la herramienta abordados anteriormente cuando exponíamos la posición de Jonas. En su descripción de la vida hay elementos para pensar la anticipación operatoria, pero esta no es su preocupación cuando habla de la herramienta como marca de la humanidad. Al mostrar la especificidad de la herramienta humana, nos remite sin nombrarla a la segunda anticipación y deja un lugar para una inteligencia abstracta. Para considerar su posición, tendríamos que evaluar por qué la reflexión, con una representación de los medios de la acción técnica, deja todavía lugar a las anticipaciones del organismo en la acción. La pregunta exige observar cómo evoluciona la práctica instrumental del hombre. La síntesis que hace Stiegler retoma de forma armoniosa las diferentes facetas del problema: la herramienta es una especie de 
memoria a través de las generaciones. La anticipación que permite la diferenciación del hombre por la de la herramienta descansa en una reapropiación de lo que las generaciones pasadas habían exteriorizado en la herramienta. Puesto que existe una real anticipación, hay más que una restitución. Se debe mostrar cómo esta anticipación integra una sensibilidad a las diferentes soluciones.

\section{LA "INORGANICIDAD" DE LA HERRAMIENTA: UNA MARCA DE HUMANIDAD ANTICIPADA}

Jonas muestra un proceso de libertad en el fenómeno de la herramienta y habla de su "inorganicidad" "12. la herramienta no tiene nada que ver con el organismo del hombre. Esto significa que la técnica humana no es hereditaria. Comprendemos esto con relación a la condición desprovista del hombre, en lo que atañe a su cuerpo y al carácter innato de su comportamiento. Así pues, la herramienta es requerida por una carencia y encuentra tanto más su lugar cuanto que un comportamiento poco determinado es al mismo tempo más flexible. ¿En qué no contribuye la carencia natural del organismo a la aparición de la herramienta? Al buscar tal espacio de causa a efecto, es preciso reconocer la apertura del organismo en lo que una búsqueda a tientas propone. Confiada al organismo, esta búsqueda no es completamente ciega. Incluso sin una verdadera representación de la acción, esta se adapta a una finalidad que es constitutiva de un ser vivo capaz de sentir una necesidad. Elegimos explotar aquí el análisis jonasiano de la vida orgánica con respecto a la acción instrumental, mientras que Jonas habría podido explicitar además cómo la libertad, que es la técnica, se enraíza en la condición fisiológica del hombre y cómo reposa en la locomoción, la percepción y la emoción. Esta perspectiva supone

${ }^{12}$ Es la traducción que Sabine Cornil y Philippe Ivernel hacen del término Organfremdheit, en (Jonas, 2000, p. 65). aprender por nuestra relación con el mundo, tal como nuestro cuerpo nos lo muestra.

La "inorganicidad" de la herramienta, como carencia del organismo, nos expondría a remitirnos a una compensación por un proceso inteligente. No ver la articulación de la herramienta al organismo, es confiscarle al organismo cualquier poder de anticipación para confiarlo a las facultades mentales. De esta manera, nos proponemos relativizar esta "inorganicidad" que lleva a una mirada exterior sobre el organismo, donde incluso la mano deviene un objeto. Jonas muestra un ejemplo de este tipo de mirada cuando, en el Principio de responsabilidad, retoma un pasaje de Aristóteles donde este se refiere a la mano como la herramienta de las herramientas. Al ir hasta el fin de esta intuición, Jonas reconoce que la mano es "la herramienta paradigmática" y que, de otra parte, la expresión "herramienta de las herramientas" concuerda con el hecho de que "las herramientas artificiales son creadas y manipuladas como su extensión" (Jonas, 1990, p. 88). ¿No es concederle al organismo un nuevo papel en la aparición de la herramienta?

Algunas interpretaciones de la herramienta van todavía más lejos en cuanto al papel del organismo. Por ejemplo, Ernst Kapp sugiere que la herramienta toma a la mano como modelo ${ }^{13}$. La mano sería de alguna manera la causa formal de la herramienta. Es necesario señalar aquí que un movimiento en el activo de un sujeto logra formar un objeto que le es exterior. Como dice Cassirer a propósito de la Philosophie de la technique de Kapp, si una herramienta primitiva es una imagen del cuerpo humano en el ser exterior,

\footnotetext{
${ }_{13}$ Para Ernst Kapp, la mano aparece en el origen de las herramientas. "La mano, articulada entre palma, pulgar y dedo, abierta, cruzada, dedos separados, tirando, cogiendo, empuñada, sola o sostenida por todo el antebrazo extendido o plegado, es la madre de todas las herramientas calificadas de acuerdo a ella como manuales". (E. Kapp, 1887; Cassirer, 1995, p. 86).
} 
se sugiere así que es la idea de una mayéutica operada por la herramienta ${ }^{14}$, donde la herramienta parece ser una especie de espejo para un individuo y una suerte
Mirar una herramienta desde el exterior no coincide con el modo de acción en que la herramienta se vuelve transparente en la intención. La contradicción se esfuma si vemos que pertenece al inicio de una etapa en la evolución de la técnica. La herramienta, que ha salido progresivamente de la acción del organismo, permite pasar a una cierta objetividad. Es necesario admitir al comienzo un fuerte vínculo de la herramienta con el organismo, o más bien con algunas libertades del organismo. Es todavía muy pronto para hacer de la herramienta un verdadero objeto respecto de quien la utiliza. Pero en el proceso que llevará a un pensamiento técnico de este objeto, el hombre encuentra así algo que le hace tomar conciencia de su acción sobre el mundo, y algo que lo orienta hacia una representación de su actuar.

En su texto sobre la herramienta, Jonas prácticamente no menciona la dimensión social del marco humano donde empieza la técnica. Esto se explica por la vía que sigue en este artículo: parte de elementos todavía observables para hablar del propio hombre. Esto hace difícil la comprensión de los fenómenos. En particular, pasa prácticamente desapercibido lo que ha aportado la vida colectiva del hombre. Estudios como el de Leroi Gourhan (Leroi Gourhan, 1957, pp. 55-79; 1964; 1971) muestran cómo al lado de la memoria biológica, entendida como la que porta el organismo (puesto que es innata), existe no solo la memoria individual cuyo soporte es el sistema nervioso, sino además la memoria colectiva de un grupo étnico. Conviene pues insistir

\footnotetext{
14 "Cualquier herramienta exterior es una imagen corporal de este tipo; en una formación material dada del mundo exterior, es la contra-prueba y el reflejo de la forma y de las proporciones del cuerpo" (E. Kapp, 1887; Cassirer, 1995, p. 86). Cassirer subraya los límites de la validez de este principio, pues la técnica pasa enseguida por una emancipación del modelo natural.
}

en el hecho de que la herramienta se retoma en una tradición técnica, en una cultura. La intencionalidad de la acción técnica se vuelve muy diferente una vez reubicada en este contexto cultural.

\section{DESPUÉS DE LA HERRAMIENTA PREHISTÓRICA}

Las preguntas planteadas por el comienzo de la técnica son cautivantes. Al remitirnos a nuestras raíces, nos remiten a lo que somos. Y permanecerá siempre en lo que hacemos en la actualidad, por ejemplo, los gestos para comprender la importancia de los automatismos. Estos gestos nos pueden servir de referencia para comprender el pasado de la técnica. Pero es necesario reconocer también cómo el organismo acoge la ayuda de artefactos basados en principios más abstractos. Sin considerar la técnica moderna en su racionalidad específica y sin entrar en las consideraciones psicológicas, económicas, ecológicas o políticas de su objeto, quisiéramos más bien limitarnos a diferentes aspectos de la técnica que se relacionan con la experiencia corporal. Algunos elementos de la historia de la técnica nos introducirán allí. No podemos olvidar el problema tan particular de las prótesis que muestran una nueva articulación de lo orgánico con lo inorgánico. Por lo demás, la reflexión se podría extender a la medicina y la farmacología.

\section{HACIA TÉCNICAS MENOS INTUITIVAS}

Durante mucho tiempo los objetos técnicos han sido el resultado de una técnica que permanecía considerablemente empírica. Por el contrario, en la actualidad muchos artefactos son el objeto de una investigación técnica importante. En muchos aparatos presentes en nuestras casas, así sean los equipos electrodomésticos más sofisticados, medios de comunicación o de informática, el principio del 
funcionamiento escapa a la mayoría de los usuarios. Interesémonos en su concepción: quisiéramos saber si aquí interviene una relación con una experiencia práctica donde el cuerpo desempeña un papel importante, en particular como coordinador de gestos en una situación dada.

De la respuesta a esta pregunta depende el interés de una extrapolación de las experiencias de nuestro examen de la herramienta prehistórica. A propósito de la talla de herramientas de piedra, la referencia corporal permite reproducir los gestos, sentir la adecuación de estos gestos y asegurar su coordinación. ¿Qué sucede con esta referencia en lo que atañe a una etapa un poco más tardía como el dominio del fuego, en tanto que este permite transformar los materiales de tal modo que un hombre no puede imaginarse hacer suyo por medio de su cuerpo? Aparecen entonces nuevas técnicas, pensemos por ejemplo en la metalurgia. Aun cuando todavía interviene una acción mecánica del cuerpo (martillado en frio, forja, etc.), aparece un poder que nada tiene que ver con esta acción del cuerpo. El mito de Prometeo sugiere que lo propio del hombre es no haber recibido un equipo directamente utilizable como garras, una piel gruesa, una poderosa mandíbula, una musculatura que especializa al hombre para correr 0 alas para volar $^{15}$, sino el fuego, como si aquí se simbolizara la técnica en su poder de cambiar la naturaleza de las cosas. Es una manera de evocar lo artificial. Debemos preguntarnos qué estatuto darle a lo artificial en la vida del hombre, cuya dimensión orgánica tenemos que respetar. Nos parece sugestivo considerar que la técnica hace parte de la vida concebida como el conjunto de la vida orgánica y de procesos inorgánicos organizados por la inteligencia del hombre (o lo que conduce a esto). Esta libertad con relación al cuerpo, más o menos grande según el carácter artificial y la referencia a la ciencia y a una memoria tecnológica, nos permitirá una relectura de la técnica moderna.

\footnotetext{
${ }^{15}$ Epimeteo distribuyó cualidades a las razas mortales. Estas cualidades, concebidas para el hombre, todavía eran del orden del organismo. Pero Prometeo robó el fuego para el hombre, que no había recibido nada, como si el fuego todavía fuera de un orden distinto con relación a las cualidades palpables de la materia, ya fuera orgánica o inorgánica.
}

\section{¿UNA CIENCIA INTUITIVA DE LO NO-INTUITIVO?}

Sería un error escindir muy brutalmente dos especies de técnicas sobre la base de sus principios de funcionamiento, ya sean intuitivos o abstractos. En efecto, estos no son del uso o de la obtención de objetos técnicos: los objetos intervienen en un comercio del ser humano con su medio, ya sea para moverse, para actuar sobre la materia 0 para permitir una extensión del ver y del comunicar. Una mirada sobre las ciencias y las técnicas nos permitirá relativizar el carácter menos intuitivo de algunos procesos explotados en la técnica. Pues a la dimensión práctica de la aplicación de un principio teórico se agrega la acusación del carácter puramente no-intuitivo de este principio.

En un primer momento, Jonas nos guiará para caracterizar el objeto de la ciencia. En una historia de la ciencia y la técnica (Jonas, 1974), recuerda que la ciencia moderna ya no reposa sobre la experiencia sino sobre la experimentación, y subraya que para la teoría la naturaleza es diferente de lo que es en su aparecer. La mirada de la física es artificial en el sentido en que nos aleja de la relación inmediata con el mundo. Nos parece que esta distancia se debe matizar: en sus trabajos, los investigadores de algún modo se familiarizan con los procesos de manera que el solo conocimiento de las leyes de la naturaleza no lo permitiría. Existe un marco teórico, pero le permite lo análogo de una percepción de la realidad y con frecuencia en lo que tiene de no-perceptible. De allí se derivan nuevos experimentos donde se adquiere una especie de intuición de los procesos. Podríamos señalar en este sentido que el conocimiento científico y técnico no descansa solamente en reglas y leyes, sino también en ejemplos que aumentan la intuición de las similitudes entre los fenómenos ${ }^{16}$.

\footnotetext{
16 Para decirlo como Thomas Kuhn en La structure des révolutions scientifiques, al hablar de algunos fenómenos físicos entre los cuales existen leyes similares, "la naturaleza y los términos se aprenden al mismo tiempo" (Kuhn, 1983, p. 260).
} 
A propósito del magnetismo o de las aplicaciones prácticas de la química, las magnitudes características no se perciben directamente por los sentidos. Pero esto no impide a quienes ven evolucionar estas magnitudes asimilar los comportamientos de la realidad por medio de su trabajo de experimentación y de tener una cierta intuición de esto. Existen analogías entre las leyes físicas que funcionan para distintos campos. Explican una asimilación más rápida, porque se presenta una cierta organización de la realidad, lo que la experiencia corporal muestra directamente por la geometría y la dinámica.

Existen buenas razones para pensar que la técnica solo avanza con el apoyo de la teoría. El progreso no solo supone un saber, sino un saber-hacer como el del factótum. Además, esto supone, para decirlo con Bertrand Hiérard-Dubreuil, que se entra en la búsqueda de soluciones como en un juego donde el conocimiento técnico hace parte de las reglas (Hiérard-Dubreuil, 1997, p. 132) ${ }^{17}$. La innovación exige sobrepasar un conocimiento basado solo en las leyes físicas: el técnico necesita una consciencia práctica y de alguna manera intuitiva de los problemas, conocimiento que se adquiere como actor en esta realidad. Sin duda, las sensaciones que aquí se experimentan no son directamente las del cuerpo, pero es importante percibir cómo el investigador, en el transcurso de sus manipulaciones, se familiariza con la evolución de los diferentes datos que las caracterizan.

\section{OBJETOS TÉCNICOS MÁS O MENOS FAMILIARES}

Desde que nuestro mundo fue invadido por artefactos de toda clase, la familiaridad con los objetos técnicos no es un hecho solo para técnicos y científicos. Jonas

\footnotetext{
${ }^{17}$ También hacemos la comparación con lo que Thomas Kuhn dijo acerca de un conocimiento tácito en la ciencia: la diferencia está entre hacer la ciencia y aprender las reglas de la ciencia (Kuhn, 1983, p.260).
}

lo subraya en un artículo de Technik, Medizin und Ethik donde nos dice que el hombre está cada vez más mecanizado o que nuestra vida está invadida cada vez más por aparatos que, no solo nos ahorran esfuerzos en el sentido físico, sino que también sirven a nuestros sentidos y espíritu (Jonas, 1985, p. 35). Nos proponemos ver por qué es crucial una especie de familiaridad con los aparatos, por el sentido de numerosos gestos de nuestra vida. Esta familiaridad descansa en el organismo. Aquí parece algo equivalente a lo que suponía la habilidad en la elaboración y el uso de las herramientas de piedra. La habilidad en la que pensamos es del orden de la acción: la herramienta se olvida y es más importante sobre todo el fin de la acción. Al comparar la talla de una piedra con la conducción de un automóvil observamos que, en ambos casos, se trata de adquirir automatismos y de construir secuencias de gestos memorizados por el cuerpo. Con el hábito se sobrepasa rápidamente el estadio del conductor que está aprendiendo. Algunos reflejos se acondicionan de tal manera que el automóvil es de cierto modo transparente. Tomamos el término transparencia de Don Ihde (Ihde, 1979; 1990), un fenomenólogo que se plantea el problema de las relaciones con los objetos técnicos. En cuanto a la trasparencia, es necesario comprender que la intención implica directamente la idea de su realización, sin que nos planteemos el problema de esta realización, ya sea a nivel de los mandos que se deben accionar o a nivel de los procedimientos técnicos que la permiten. Así pues, lo que queda es una relación directa del organismo con el vehículo. Sería una lástima ver directamente un remplazo de la acción del organismo por el funcionamiento del artefacto: los esfuerzos físicos ahorrados no impiden una cooperación y una asimilación de este funcionamiento en la acción de una persona. Pensemos en un vistazo, es decir, en una especie de juicio implícito, que nos permite evaluar si podemos estacionarnos en un lugar no obstante muy pequeño, un poco como si hubiéramos insertado 
nuestro cuerpo en las dimensiones del vehículo que conducimos. Se trata de una especie de incorporación, para retomar el vocabulario de Don Ihde.

Señalamos también, al utilizar medios técnicos muy complejos, algunas de sus características: pensemos en las reacciones de conjunto de un automóvil. Se trata de reacciones de alteridad. Por ejemplo, diremos que un motor es brutal, que se ahoga, que es nervioso o que es suave y dócil. En este caso, se hablará del temperamento o del carácter de un automóvil. La técnica nos procura mediadores para la acción al darnos especies de prolongaciones de nosotros mismos, ya sea para trabajar o para movernos, y apreciamos de diversa forma lo que llega a reforzar 0 completar la acción. Y luego, esta mediación se vuelve habitual y su carácter solo aparece si algo obliga a plantear la cuestión.

\section{LOS ARTEFACTOS, PROLONGACIONES DEL ORGANISMO REINTEGRADOS A LA VIDA}

En la acción técnica, el hombre como organismo se halla frente a artefactos igualmente organizados $y$, a causa de esta organización, reacciona como si se encontrara frente a una especie de viviente artificial. Pensemos aquí en la electrónica que permite controlar el funcionamiento de un aparato que, a su vez, permite nuevas operaciones a nivel de la información y la comunicación. De este modo, al sobrepasar una especie de animación mecánica de los artefactos, la técnica moderna puede volverse más familiar, a menudo por una suerte de inteligencia otorgada a los artefactos, como si de algún modo los hubiéramos humanizado. Estos equipos están frente a nosotros, pero sabemos que en la práctica, una cierta facilidad en el uso, ergonomía y convivialidad nos permiten asimilarlos en nuestra acción.
Las relaciones de transparencia y alteridad no son las únicas que se pueden reconocer en nuestras relaciones con los objetos técnicos, pero son las más importantes en lo que compete a una relación con el organismo. En el enfoque del objeto técnico, estas relaciones incluyen la alternativa ya mencionada a propósito de la herramienta prehistórica entre el modo de la acción que supone una transparencia del artefacto y una percepción de la alteridad del ser de la herramienta. Naturalmente, la elección de las opciones depende también por una parte del tipo de objeto. Esta alternativa es una clave de lectura para articular la percepción de la técnica en la actualidad. Además, la clasificación según diferentes tipos de relación reactiva el problema de la noción de técnica. De un lado, es un proceso de libertad por medio de la cual el hombre se libera del dominio de la vida (aun cuando este proceso apunte a satisfacer las necesidades orgánicas) y la herramienta (en el sentido genérico del término) es entonces lo que marca esta diferencia. Pero otra posibilidad es verla como una apertura de un ser vivo que toma una herramienta. Al ir todavía más lejos, sería también, de una manera más general, una potencialidad de la vida para producir las primeras formas de la herramienta y reorganizar la acción para dar lugar al uso de la herramienta, que progresivamente deviene objeto de la cultura, en la relación con el mundo que es la vida. Encontramos aquí dos miradas que se aplican a la técnica desde sus comienzos hasta nosotros, cualquiera que sea la complejidad de los principios del funcionamiento. En un caso, observamos la técnica desde el exterior. Su carácter artificial contrasta con la naturaleza. Así pues, ignoramos un cierto conocimiento de la técnica que está disponible para nosotros, en la otra manera de ver, como desde el interior de la práctica. A partir de este segundo punto de vista, el carácter técnico se borra en la acción. Los objetos técnicos se vuelven, querámoslo o no, una especie de medio de vida. Intervienen como 
mediaciones en nuestras representaciones y como prolongaciones de nosotros mismos.

\section{PROLONGACIONES DE LA ACCIÓN Y DEL ORGANISMO}

La técnica no hace otra cosa que reforzar y diversificar nuestra acción, de una manera más o menos asimilada por el cuerpo. También provee medios para preservar la salud y la integridad de un organismo. Pensemos en las prótesis. Algunos tipos de prótesis muestran cómo un miembro, de cierto modo, no es tan diferente de una herramienta artificial. De esta manera, la medicina se funda radicalmente en la relación de la técnica con el organismo. Se constata una preocupación por perfeccionar sin cesar lo que podrían ser los substitutos de lo natural, e incluso usar la materia viviente para producir nuevos artefactos que estén compuestos de lo artificial y natural.

Por lo demás, observamos una consecuencia de la técnica sobre nuestros cuerpos que está en la continuidad de la noción de prótesis y que sobrepasa el estricto marco de un problema de salud. Al tomar el lugar del hombre en las tareas difíciles, en los trabajos que no habría podido hacer por falta de fuerza, la técnica ha disminuido, de manera general, el entrenamiento de un cuerpo ya desprovisto relativamente desde el punto de vista físico. ¿Lo que prolonga nuestra acción en el mundo, no es del orden de la prótesis desde el momento en que no podemos prescindir de esta para vivir una existencia, por lo demás reformada por la técnica? Esta dependencia, ligada a toda la organización económica de la vida, se parece a una falta de libertad, aun cuando no aparece como tal cuando el objetivo que se persigue es la salud y la vida de las personas, como en la medicina. En este caso, lo que se ve como una asimilación de lo que es extraño, relativiza el carácter de prótesis. Es preciso discernir si la medicina conserva realmente el mismo objetivo a través de una práctica que ha variado de manera sensible a causa de los medios que se le ofrecen, y donde a menudo las dimensiones técnicas y humanas no están bien equilibradas. Esto se podría evaluar en las diferentes prácticas médicas como el diagnóstico, las operaciones quirúrgicas o los tratamientos. Lo que también se presta para interpretaciones filosóficas y, en particular, para consideraciones éticas. La parte que reposa en la técnica a nivel del diagnóstico permite dar informaciones más precisas, pero informaciones más técnicas, que tal vez olvidan que el hombre solo es un organismo. Puesto que es un organismo humano, ya es una persona y no una máquina con piezas más o menos fiables. El objetivo de tratar el cuerpo se volvería muy pronto el de mejorar el cuerpo. Incluso sin pregonar este ideal, es un mensaje que se arriesga a crear una especie de utopía donde se niegan los limites humanos. ¿Esto no se ilustra por el fenómeno del dopaje, donde sobrepasar las marcas humanas se sanciona como una trampa, cuando ha sido posible por una preparación que le plantea a un deportista el problema de la verdad de su contribución en estas marcas?

\section{CONCLUSIÓN}

La técnica nos abre nuevas posibilidades para nuestros movimientos, para hallar alimento, amplía nuestra manera de ver, entender, comunicar y curarnos. También tiene consecuencias en la manera en que formamos un proyecto de vida e induce una cierta lógica en la relación entre un deseo y su realización. $\mathrm{Su}$ complejidad oculta algunas veces los medios de su acción, aun cuando estos se asimilen en una nueva manera de actuar. ¿Nos ofrece una explicación el rodeo por la filosofía de Jonas y por el problema de la herramienta? La discusión sobre la aparición de una inteligencia técnica a través de las prácticas instrumentales ofrece otra perspectiva sobre la racionalidad técnica, difícilmente pensable sin una relación con la práctica. Este vínculo con la práctica reubica la técnica en lo que hace la vida. Es importante 
señalarlo en el momento en que algunos objetos técnicos cobran una importancia desmesurada en el imaginario del hombre.

La técnica no se volverá un fin en sí si sacamos algunas conclusiones de la manera por la cual, para la herramienta, el organismo se encargaba de organizar la vida dejando un lugar a lo inorgánico. Jonas nos ha mostrado esta manera de ver la vida, pero sin articular la técnica tanto como la esperábamos. La técnica exige una nueva manera de vivir que toma mejor en cuenta lo que es. De alguna manera, es preciso comprender que los dos posibles enfoques de la técnica (desde el exterior por la teoría o desde el interior por la práctica) son complementarios y de hecho se juntan. El primero, nos dejará medir las oportunidades y los riesgos para hacerla entrar en un proyecto de sociedad y, el segundo, nos ayudará a vivir mejor en relación con la técnica en este proyecto.

\section{BIBLIOGRAFÍA}

Cassirer, E. (1972). Philosophie des formes symboliques, La pensée mythique, París, Éditions de Minuit, t. II. [(1998) Filosofía de las formas simbólicas. El pensamiento mítico, México, F.C.E., t. 2].

Hiérard-Dubreuil, B. (1997). Imaginaire technique et éthique sociale, Bruselas, De Boeck.

Ihde, D. (1979). Technics and Praxis, A Philosophy of Technology, Boston-Londres Reidel Publishing Company, Dordrecht.

(1990). Technology and the Lifeworld, From Garden to Earth. Bloomingtown-Indianapolis, Indiana University Press.

Ingold, T. (1983). "Tool-Use, sociality and intelligence", en Tools, Language and Cognition in human Evolution, Kathleen R. Gibson y Tim Ingold ed.,
New York, Cambridge University Press.

Jonas, H. (1987). "Warum die moderne Technik ein Gegenstand fur die Philosophie ist”, en Technik, Medizin und Ethik, Francfort sur le Main, Suhrkamp.

(1993). "Technik, Freiheit und Pflicht" en Dem bösen Ende näher. Gespräche über das Verhältnis der Menschen zur Natur, Suhrkamp, Frankfurt).

(1974). "Seventeenth Century and After: the Meaning of the Scientific and Technological Revolution", en Philosophical Essays. From Ancient Creed to Technological Man, Englewood Cliffs, Prentice-Hall.

(1963). "The Nobility of Sight" en The Phenomenon of Life. Toward a Philosophical Biology, New York, Harper and Row.

(1963). The Phenomenon of Life. Toward a Philosophical Biology, New York, Harper and Row.

(1990). Le Principe responsabilité. Essai d'une éthique pour la civilisation technologique, [(2004). El principio de responsabilidad: ensayo de una ética para la civilización tecnológica, Barcelona, Herder].

(2000). "Outil, image et tombeau. Du transanimal dans l'humain", artículo retomado en Évolution et liberté, traducción de Ph. Ivernel y S. Cornil, París, Rivages.

Kapp, E. (1887; 1995). Grundlinien einer Philosopbie der Technik, Braunschweig, citado por Cassirer en "Forme et technique", en Écrits sur l'art, París, Cerf).

Kempf, H. (1997). "La naissance des outils", en $L a$ Recherche $n^{\circ} 296$. Especial "L'histoire de la vie. Trois milliards d'années d'évolution", París. 
Kuhn, T. (1983). La structure des revolutions scientifiques, París, Flammarion. [(1971). La estructura de las revoluciones científicas, México, F.C.E.].

Leroi-Gourhan, A. (1971). Evolution et Techniques, Milieu et Techniques, París, Albin Michel, t.2. [(1989). Evolución y técnica; El medio y la técnica, Madrid, Taurus, t. 2].

(1957), "Le comportement technique chez l'homme et chez l'animal", en L'évolution bumaine, Spéciation et relation, París, Flammarion.

(1964). Le geste et la parole, La mémoire et les rythmes, París, Albin Michel, t. 2. [(1971). El gesto y la palabra, Caracas, Universidad central de Venezuela].

(1966). Le geste et la parole, Technique et langage, París, Albin- Michel, t.1. [(1971). El gesto y la palabra, Caracas, Universidad central de Venezuela].
Piveteau, J. (1962). L'origine de l'homme. L'bomme et son passé, París, Hachette. [(1962). El origen del hombre, Buenos Aires, Hachette].

Ricoeur, P. (1967). Philosophie de la volonté, Le volontaire et l'involontaire, París, Aubier Montaigne, tomo I.

Stiegler, B. (1994). La technique et le temps, La faute d'Épiméthée, París, Galilée, vol. I. [(2002). La técnica y el tiempo, El pecado de Epimeteo", Hondarribi (Guipuzkoa), Hiru, tomo I.

Tinland, Fr. (1977). La différence anthropologique. Essai sur les rapports de la nature et de l'artifice, París, Aubier Montaigne.

Wynn, T. (1983). "Layers of Thinking in tool behavior", en Tools, Language and Cognition in buman Evolution, Kathleen R. Gibson y Tim Ingold ed., New York, Cambridge University Press. 